\title{
Challenges and Opportunities of Using Digital Storytelling as a Trauma Narrative Intervention for Children
}

\author{
Kim M. Anderson \\ Jonathan R. Cook
}

\begin{abstract}
This article address the challenges and opportunities of implementing a webbased Digital Storytelling (DS) curriculum to supplement the trauma narrative component of Trauma-Focused Cognitive Behavioral Therapy (TF-CBT) for traumatized young people, ages 9-17, receiving mental health services at a rural domestic violence (DV) agency. Digital storytelling, as the term suggests, combines storytelling with technology that integrates a mixture of digital images, text, audio narration, and music. Ultimately, implementing the DS curriculum empowered participants to process and develop their trauma narratives in a multi-sensory, accessible, and coherent manner. In doing so, they gained tools (writing, narrating, illustrating, and ultimately assembling their own stories) to form adaptive responses regarding their family violence experiences in the immediate aftermath and possibly over time. Agency implications are discussed regarding training, technical, and confidentiality issues related to the implementation of a web-based DS curriculum.
\end{abstract}

Keywords: Digital storytelling, narrative, children exposed to domestic violence, traumafocused cognitive behavioral therapy

This article focuses on the use of digital storytelling (DS) as a narrative intervention for traumatized young people, ages 9-17, receiving mental health services at a rural domestic and sexual violence agency. In particular, we developed a web-based DS curriculum to supplement the trauma narrative component of Trauma-Focused Cognitive Behavioral Therapy (TF-CBT), an evidence-based intervention for children with emotional and behavioral difficulties associated with violence exposure and trauma. TF-CBT is typically delivered in 12-16, weekly 90 minute sessions (60 minutes with the child, 30 minutes with the parent to recap the child's session). The core components of TF-CBT make up the acronym PRACTICE: psycho-education, relaxation skills, affect modulation, cognitive coping, trauma narrative development, in-vivo exposure, conjoint parent-child meeting, and enhancing safety (Cohen, Mannarino, \& Deblinger, 2006).

We sought to understand the elements (i.e., digital images, text, audio narration, and music) of DS and their individual and collective narrative and mental health benefits (Gubrium, 2009; Lambert, 2006). DS was intuitively recognized by agency staff as a good fit with the cognitive, emotional, and linguistic needs of children, yet it was a novel intervention. Therefore, we chose not to use it as a stand-alone protocol and instead integrated it into the existing TF-CBT protocol. The DS curriculum is presented, along with a discussion regarding the challenges and opportunities of implementing it as a narrative intervention for youth traumatized by family violence (e.g., domestic violence, child sexual abuse/violence).

Kim M. Anderson, Ph.D., LCSW, is an Associate Professor in the School of Social Work at the University of Missouri.

Jonathan R. Cook, Ph.D., is an Assistant Professor of Psychology, Rhodes College.

Copyright (C) 2015 Advances in Social Work Vol. 16 No. 1 (Spring 2015), 78-89 


\section{Children's Trauma Narrative Construction}

Processing family violence experiences is complicated as children may repress one or more aspects, perceptions, or feelings. This chaos of fragments is difficult to understand and is associated with confounding emotions and negative appraisals of self and others, thus overwhelming children with experiences they cannot process and, consequently, inhibiting their ability to construct a coherent self-narrative (Simon, Feiring, \& McElroy, 2010). Processing, organizing, and integrating traumatic memories is challenging due to encoding in a dissociated and often sensory rather than a verbal manner; thus, they cannot be effectively communicated or organized (Niederhoffer \& Pennebaker, 2009). Integrated functions such as cognition, memory, and emotion become disconnected, particularly in cases of prolonged exposure to interpersonal trauma such as with domestic violence or child sexual abuse (Simon et al., 2010). Failure to adequately integrate trauma-related information underlies several psychological and physical problems including posttraumatic stress disorder (PTSD), low self-esteem, and destructive coping mechanisms (Cohen et al., 2006; Dube, Anda, Felitti, Edwards, \& Williamson, 2002).

Creating a written trauma narrative is a critical component of standard TF-CBT, and general guidelines are provided in a published treatment manual (Cohen et al., 2006). During the trauma narrative component, the child develops a storybook (using words, pictures, and/or drawings) or uses other creative expressions (e.g., poetry, song, art techniques) to tell the story of their trauma (Cohen et al., 2006; Cohen, Mannarino, \& Iyengar, 2011). The goal of the trauma narrative is to disentangle unpleasant associations between reminders, thoughts, and discussion of the trauma from overpowering emotions, such as horror, fear, or helplessness. Thus, over the course of several sessions, children are encouraged to write in increasing detail about the trauma, including their thoughts and feelings about it. In our experience, we found that young people often avoided the trauma narrative component because of the nature of the content and difficulty with speaking or writing plainly about their childhood trauma, as the experiences were often fragmented, difficult to understand, and associated with overwhelming emotions. We believed that digital storytelling might help them find another avenue to discover their voice during the development of the trauma narrative because of its multi-sensory nature and cultural congruence with young people's connection to digital media. Thus, we added digital storytelling to the trauma narrative component of TF-CBT, as a child-friendly, multi-media narrative alternative that integrated images, narration, and music to tell one's trauma narrative.

\section{Digital Storytelling as a Narrative Intervention}

Digital storytelling, as the term suggests, combines storytelling with technology (Lambert, 2006). DS involves a computer-based program that integrates a mixture of digital images, text, audio narration, and music. Such modalities are highly customizable, interactive, and allow the user to control how individual digital media elements interact with one another. In addition, the user can personalize digital stories with pan and zoom effects that create a sense of movement and highlight areas of importance in an image. The process of DS involves the individual assessing and making decisions regarding how the story interacts with the images, narration, or music/sounds (Gubrium, 2009). The technical 
process is user-friendly; both youth and adults often catch on quickly (Davis \& Weinshenker, 2012). Once the story is created and saved, one can view, edit, and continue to add to it until the final version is completed. After completion, all pictures, narrations, and music are compiled and exported into a single video file that one can then view in increments or in its entirety. The end result is the creation of a two to five minute film of one’s story (Gubrium, 2009; Lambert, 2006).

Traumatic memories are more disorganized than other memories, and narrative interventions aimed at organizing memory may be particularly effective for children because more organized memories are easier to integrate into existing ones (Foa \& Rauch, 2004; Hanney \& Kozlowska, 2002). Digital storytelling allows for gradual exposure to one's life traumatic experiences through the use of storyboards-a means of planning out, creating, and telling one's story in a compelling and emotionally engaging form (Lambert, 2006). DS embodies the narrative approach of “emplotment," which involves arranging temporal elements into a whole by connecting them together and directing them toward a conclusion or ending (Polkinghorne, 1991). Essentially, storyboarding transforms a sequence of disconnected images juxtaposed with narration into a unified story with a point or a theme (Anderson, 2010). For traumatized youth, DS provides a good balance between giving a basic structure to construct one's story that allows immersion into story material (e.g., family pictures) while also providing disengagement opportunities as the child learns technical tools for movie making. Such balance allows them to shift between reexperiencing negative memories while developing mastery over the traumatic experiences and its portrayal in their narrative. Consequently, traumatic reactions that young people otherwise might carry with them throughout their lives may be prevented, lessened, or ameliorated.

\section{Rural DV Agency and Case Example}

The DS curriculum outlined below developed out of the TF-CBT services offered by the only victim service provider for a rural county (population approximately 43,000) in a Midwestern state. The youth therapy program (YTP) was comprised of the first author as clinical coordinator, the second author as program coordinator, and a licensed professional play therapist. In response to the difficulty young people were having in constructing traditional written trauma narratives, a DS web-based curriculum was created and added to the trauma narrative component of PRACTICE. We wanted to better understand the capacity of DS to facilitate recovery and promote resiliency in clients, ages 9-17, exposed to familial domestic and/or child sexual violence who were experiencing post-traumatic stress disorder (PTSD). Of 41 agency referrals, 28 met criteria for PTSD and 25 of those followed through after intake to begin DS within the context of TF-CBT. Of the 25 who started TF-CBT services, 16 (9 male and 7 female, all Caucasian, ranging in age from 716 years old, $M=12 ; S D=3.28)$ completed treatment $(63 \%)$. Non-completion $(n=9)$ was most often due to life crises such as loss of housing, transportation, and/or employment.

We modified the protocol for DS from a 3-day standard workshop model for community participants (see Center for Digital Storytelling, n.d.) to one integrated into an evidence-based therapeutic model (TF-CBT) for young people with PTSD. This adaptation resulted in a web-based DS curriculum for the trauma narrative component (see Digital 
Storytelling, 2015) that provided direction and guidelines that were not sufficiently delineated in the professional literature regarding trauma story construction with children. The DS curriculum consisted of three structured sessions covering the purpose, process, and technical tools of DS and two to three unstructured sessions for them to complete their digital stories. The DS curriculum added essential structure to the trauma narrative component of PRACTICE, and it helped the children process and develop their stories of family violence in a less threatening and more approachable manner.

Initially, we used a personal computer program (Windows Moviemaker); eventually, an iPad was used (applications: Pinnacle studio, iMovie) as it was more user-friendly for participants and less costly for the agency. The clinician provided children with technical assistance and encouraged them to use their TF-CBT skills. Embedded throughout the DS curriculum were digital story examples (downloaded from public sites) of children who had survived, persevered, and even thrived despite adversity (e.g., parental death, domestic violence, alcoholism).

The first DS session began with the rationale for making a trauma narrative: for young people to tell their stories and say the things they needed to express, even if they did not know how to do so at the moment. The concept of DS was introduced by explaining that participants would create a brief movie about their lives and the difficult experiences (i.e., family violence) they had endured. They were told they would take on several roles: author, narrator, director, and editor. They were encouraged to write scripts or condensed (i.e., 100250 words) versions of their life stories. The script-development process addressed components of narrative therapy and resilience theory, along with elements of storytelling to guide each youth's script. Their scripts highlighted the important people and events in their lives, including experiences of family domestic and sexual violence, along with their strengths, coping skills, and ways of healing despite enduring adversity. Although participants were given a good deal of latitude in content, the DS curriculum provided a guide to create coherent narratives that had beginnings, middles, and ends.

In DS session two, young people finished their scripts and were introduced to how images, narration, and music/sounds would be used to complete their stories. Participants were encouraged to use personal artifacts (e.g., pictures, drawings) that could be scanned and digitized. As part of the DS curriculum, several pictures of various people, places, and landscapes were provided to help them identify associated emotions and convey how text (e.g., the word sad) was not always needed to distinguish such feelings. Different types of music were added to these pictures, and participants were asked to notice if their emotions changed as a result. In doing so, music was highlighted as another medium to convey emotion. Next, they were shown a DS of a young person's experiences with bipolar disorder and the loss of his supportive father; upon viewing it, they were asked to identify the emotions expressed through the pictures and music represented in his story.

In DS session three, participants further explored their difficult experiences (i.e., family, domestic, and/or sexual violence); the terms "difficult experiences" or "hard times" resonated more with them than the word "trauma." They viewed a digital story of a youth who used rap to tell his experiences of witnessing his father harming his mother. The clinician reiterated that the youth did not have to relive scary experiences; he/she had 
control over portraying as much or as little of his/her adversity in their DS. If participants seemed stuck, they were encouraged to focus on the end of their stories - where they were in the present moment- and to think about how they got there. To further underscore the concept of resilience, participants watched a DS in which a youth used music to cope with family alcoholism. This allowed them to see how difficult experiences were a part of their stories but did not have to define them.

In DS session three, participants also created audio-recordings of their scripts. Although some young people were hesitant, gradually they became more accepting of their voices as they read and reread their scripts aloud. For an example of the use of one's voice, participants viewed an animated DS of a youth with Asperger's Syndrome interviewing his mother about being his parent. As they audio-recorded their scripts, they played back the recordings to make changes as they saw fit. They were encouraged by the clinician to consider that their audio recordings did not have to be perfect; in other words, it was okay to hesitate, get choked up, or pause. After finishing their audio recordings, participants were asked to collect pictures (a maximum of 15) related to their script to bring in for their next session. The clinician helped them focus on the overall purpose of their stories and how one unique picture was not needed to match each word, sentence, or paragraph. In fact, a digital story could include as few as one or two pictures to accompany the narration. They were also asked to bring in music/sounds (a maximum of three) to complement their narration. The agency also had an iTunes account for participants without access to music. Instrumental music, as a background soundtrack for the audio recordings, often worked best as it did not compete with or override the audio narratives. Also, the use of silence was introduced as a compelling way to emphasize the visual narratives.

During subsequent DS sessions, participants worked on finalizing their digital stories as they interacted with different images, narratives, and background soundtracks, including decisions regarding timing and placement, allowing for as much immersion and disengagement of story material as necessary. Upon completion of the digital stories, the audio recordings, images, and music were exported into brief videos. The youth often expressed enthusiasm and pride in their finished work. Working with digital media allowed them to more easily approach their difficult family experiences than what we had observed in our previous attempts of addressing the "T" (e.g., trauma narrative) aspect of PRACTICE through the written format only. The use of a multi-sensory approach (as opposed to writing/drawing only) allowed these young people to engage in an interactive dynamic process that ultimately allowed them to use technical tools to create their stories while controlling how they were expressed. In addition to script development, other DS elements - voiceover, images, sound track - drew on their own inherent semiotic "grammar" (Kress, 2003) to expand upon the storytelling process.

As with all aspects of PRACTICE in TF-CBT, the child's digital story sessions were also shared with the non-abusing parent/guardian with the youth's understanding and consent (Foster, 2014). Caregivers often assisted them with collecting pictures and music for their DS, and observing its construction helped prepare the young people to view it in its entirety at the conjoint session (i.e., when the child and parent view the finished digital story together). The conjoint session, an important component of TF-CBT, was often powerful as parents came to new understandings and appreciation of their children's 
trauma, recovery, and resilience. As one parent stated in regard to her daughter's digital story, "The recorded voice of a young person with such a big story has a huge impact." Upon completion of TF-CBT services, each participant was given a DVD of his/her digital story. During the final session, the clinician, participants, and parents discussed guidelines, particularly safety and confidentiality issues, regarding the sharing of one's video with others.

The use of DS as a trauma narrative intervention was well-received by all participants $(N=16)$, and allowed them to process very difficult experiences in a manner that offered them creative control and various ways to portray their stories. During exit interviews, participants completed a Likert-type questionnaire asking about the process and outcome of DS in the framework of TF-CBT. These young people found that creating a digital story that included their most difficult, along with their more positive, experiences was an enjoyable process even though the material was challenging. Hearing their voice (reading their story aloud and recording their story) became an integral part of their trauma narrative process. They reported valuing the creative process along with having control over the story they wanted to tell. Participants also completed standardized instruments on PTSD, depression, self-esteem, and coping at pre- (intake session) and post-intervention (exit interview); results indicated a clinically significant difference in psychological distress and well-being over the course of treatment for all participants. See Anderson and Wallace (2015) for additional information regarding our research design and findings.

\section{Case Example: Mary}

A case example of a nine-year-old Caucasian female, pseudonym "Mary," is presented to highlight how the DS process facilitated her recovery from sibling incest. Mary resided with her 40-year-old stepfather and 34-year-old biological mother, both of whom brought her to weekly TF-CBT sessions. Approximately nine months prior to initiating agency services, Mary disclosed to her mother that her biological brother (age 13) and stepbrother (age 12) had repeatedly sexually abused her. Both boys were removed from the home and placed in an inpatient facility for sex offenders. As a result of these experiences, Mary's parents reported that she was emotionally distressed, withdrawn, and feared reminders of her brothers and the abuse (e.g., avoiding parents' bedroom where she was abused). Mary's mother reported, "My biggest fear is that my daughter will never be okay again."

During the intake process for services, Mary and her parents were introduced to the components of TF-CBT, including how Mary would use DS to tell her story of the abuse. She was shown the iPad and the DS application and was very excited about using it. In the first four sessions, Mary developed mastery of introductory TF-CBT components (i.e., psycho-education on trauma, relaxation methods, affect modulation, and cognitive coping skills). She also spent a few minutes on the iPad at the end of each session playing video games and exploring the DS program. Mary then began six consecutive sessions creating her digital story. Throughout the DS process, Mary viewed videos created by children who had faced adversity presented in the DS curriculum. In doing so, she learned how others used specific DS elements such as voice, photographs, sound, music, and pacing to tell their stories. 
During the first and second DS sessions, Mary finished her storyboard and began to write her script. Mary's storyboard included the most meaningful events in her life before, during, and after the abuse took place (a narrative with a beginning, middle, and end). She chose to first write about the happiest times in her early life followed by the abuse itself including its emotional impact, her personal strengths and resiliency capacities, and how she had learned to cope after the abuse. She wrote about the abuse and the embarrassment, fear, worry, anger, and guilt that she felt regarding it. The clinician and Mary further reviewed the DS curriculum regarding how to represent emotion and discussed how she could use specific photographs in her DS trauma narrative to illustrate particular feelings or experiences related to the abuse rather than verbally labeling the emotion. As part of the script development, Mary identified a previously unverbalized dysfunctional belief-that she was to blame for the abuse and the subsequent removal of her brothers from their home. Her challenging this belief, with the support of the clinician and her parents, became an important aspect of her therapeutic process.

In the third DS session, Mary completed her script and narrated it. Listening to her recorded story was a powerful and emotional experience for Mary. Initially, she described listening to her own voice as "really weird." After listening to herself several times, she stated that she liked it, especially the narrated ending: "I'm learning to be okay even though I was abused. I can think about the abuse and talk about it and cope and not be as upset as I used to be. I feel happy. I feel safe. I feel loved. My parents are there for me.” The audiorecording process provided Mary with some distance from the abuse while simultaneously empowering her through the control she had narrating her own story. Her voice became louder, stronger, and less tentative towards the narration of the abuse she experienced.

During the fourth through sixth DS sessions, Mary brought in family photographs that she had selected with the assistance of her parents and sequenced them to her audiorecording. She chose photographs based on literal rather than abstract meanings (i.e., showing a picture of her home when describing what it was like growing up there). She sequenced the images chronologically according to the specific events she described in her narration (i.e., growing up, the abuse, and current coping/resiliency). Next, she assembled the content using the iPad.

Mary was particularly excited about this stage of the DS process because she was excited to be working on an iPad, and the technical process appeared to be intuitive for her. Mary chose to not use background music and instead let her voice be the focal point. After she completed the assembly of digital media, it was exported as a video file.

Implementing DS specifically for the trauma narrative aspect of TF-CBT enabled Mary to negotiate a balance between representing her traumatic experiences with her words and pictures while simultaneously learning technological skills. By the time Mary viewed the final exported video, she had listened to the audio and her digital story more than a dozen times. Initially, Mary found it difficult to watch and listen to the DS, particularly during the sequence where the photographs of her brothers were paired with her narration of the abuse. However, with repeated viewings Mary was able to watch the entire digital story with minimal or no distress due to desensitization during the DS process. When the clinician asked Mary about this change she said, “I'm learning how to cope with the hard 
times I've been through.” Through multiple forms of repeated exposure embedded in the development of the DS trauma narrative, Mary's distress decreased (as in all aspects of PRACTICE, participants are asked to rate their distress on a Subjective Units of Distress of Scale, SUDS) and her mastery of technical and storytelling increased as indicated in her coherent, integrated, and completed story.

Throughout the creation of Mary's digital story, the clinician shared it with her parents (without Mary present but with her knowledge and consent) so they could process their own feelings and reactions about the abuse as well as have a sense of her narrative progress. Initially, both parents found listening to and viewing the digital story difficult, particularly the sections in which Mary described the abuse. During these meetings, the clinician talked to Mary's parents about their feelings of guilt and responsibility regarding the abuse, as well as how they could support their daughter's coping and resiliency abilities. Similar to Mary's own experience, her parents found the digital story less distressing with repeated exposures. Ultimately, they came to view Mary's concluding comments on her coping, resiliency, and hope for the future as an especially powerful component of her story.

Next, a conjoint parent-child session was held with the clinician to view the completed digital story together. This was an emotional but positive experience for both Mary and her parents, who expressed their love and support for her. They also discussed the positive changes they had seen Mary make over the course of treatment. The in vivo (e.g., in-session desensitization to trauma stimuli) component of PRACTICE ended up not being necessary as DS construction exposed Mary to triggers, such as pictures of her abusers and the location of the abuse, to which she became desensitized. Upon completion of TF-CBT services, Mary and her parents were given a DVD of her digital story, and guidelines regarding the sharing of her story, particularly safety, legal, and confidentiality issues, were addressed.

\section{Discussion and Implications}

Several studies demonstrate the relation between formulating an integrated and coherent story after experiencing traumatic events and enhanced trauma recovery and growth (e.g., Anderson \& Hiersteiner, 2008; van Minnen, Wessel, Dijkstra, \& Roelofs, 2002). The organization of stressful memories, particularly in a narrative structure, is a critical factor in the beneficial effects of children processing their stories about stressful events (Hanney \& Kozlowska, 2002; Simon et al., 2010). When children process the story of their family violence experiences in depth and detail, their recent and traumatic memories are transformed and more easily incorporated into their life narratives. DS empowers children to address difficult experiences in a deliberate manner while giving them creative control and a variety of ways to tell the story. The sequential process of script development, audio-recorded self-narrative, selection of photographs, and assembly of the video incorporates multiple sensory modalities (i.e., visual, auditory, tactile) to help youth tell one's story in manageable doses. Such processing allows children to elaborate and organize their trauma memories while tolerating the negative emotions associated with it. Our research findings indicate that being able to gain insight and understanding into a personally traumatic event for these participants enhanced feelings of efficacy and control over the event, one's emotions, and life in general (Anderson \& Wallace, 2015). 
DS as a trauma narrative intervention provides young people with a structured, interactive, and empowering method of processing traumatic experiences. We were amazed by the facility with which participants learned and developed digital storytelling skills while processing their traumatic life experiences. We found that they were more willing to revisit the past and address present emotions than they had been with the written narrative as they had the structure and tools to regulate exposure to trauma material. Participants often addressed their difficult experiences with interest rather than avoidance even though they experienced discomfort regarding abuse-related memories, emotions, and cognitions. As in the example of Mary's case, the inclusion of her voice and family photographs was initially distressing, but she quickly habituated to these new stimuli and no longer found them to be upsetting. DS provided a good balance between giving a basic structure to construct her story that allowed immersion into story material (e.g., family pictures) while also providing disengagement opportunities as Mary learned technical tools for movie making. Despite the seriousness of the abuse, Mary fully participated and even came to enjoy working on her story, particularly when it came time to use the iPad. Working on a tablet-sized device may also have increased Mary's feelings of self-efficacy because she could sit comfortably and hold it in her lap as opposed to having to work at a table and use a larger desktop computer.

The addition of a web-based DS curriculum in the context of TF-CBT provides mental health clinicians with practical information on narrative development to use in their own practices. The clinician's role is to provide a safe environment for each youth to develop his/her digital story and to support the process. Because of the way trauma affects children's processing, their story creation can appear random and non-linear at first. However, over time they can often create meaningful and profound stories about their experiences. We found that young people have an intuitive sense of how they want their stories to develop. They knew how they wanted their stories portrayed in regard to the sequencing of images, music selected, and the use of their voice, even though they may not have been able to articulate the "why" and "how" of their process. Consequently, we learned that it was best to not inquire about the construction process, e.g., asking them to talk about what their digital images represent. Instead, it was better to focus on the written script and ask questions regarding how selected images, songs, etc., helped to complete their stories.

A few areas to consider before clinicians adopt DS as a trauma narrative intervention include training, technical, and confidentiality issues. In addition to staff being educated on TF-CBT, the first author, who was trained at the Center for Digital Storytelling, also provided instruction and guidance on digital storytelling. Although our DS curriculum is a useful tool for clinicians, it may not be sufficient for their technological needs without oversight from someone skilled in DS. In addition, agencies will need internet access and personal computers with moviemaking programs. If iPads are used, the applications are relatively inexpensive (e.g., \$5.00); however, the agency still needs to have a personal computer to download and burn the video to a DVD. Also, technological failures may result in the loss of digital data gathered during the trauma narrative sessions. For example, in Mary's case, her voice recording was unusable as the audio file was corrupted. Fortunately, Mary was good-natured about re-recording the narrative, but this could be upsetting for 
some clients. Using a built-in audio recorder found on most smartphones and subsequently importing the file onto the iPad might be one potential solution to avoiding lost data. Furthermore, teaching clients how to use the software can constitute an additional time commitment, especially for less technologically savvy clients. However, as younger generations are raised in increasingly technologically immersive environments, the need for such training may be minimal.

Confidentiality guidelines must be considered when using any electronic device to collect and assemble digital stories. A portable device containing DS materials should be password protected and not include the clinician's personal photographs or videos. In addition, an electronic album of photos should be created for each client to prevent clients from seeing the images of others. Agency administrators will also need to make decisions on how the videos will be stored upon termination of services and if clients will receive a copy of their completed stories. The DV agency for the current project, after much staff discussion, decided that clients had a right to their digital stories and provided them with copies. However, the clinician met with all participants and caregivers to discuss the implications of having their videos, including possible legal and confidentiality ramifications. Although implementation of DS requires careful agency consideration, the benefits include a powerful, highly personal, and multisensory method for clients to process traumatic events.

\section{Conclusion}

Trauma is a deeply personal experience, and no one child will tell his/her story the same. Narrative approaches, such as digital storytelling, can influence trauma recovery and resilience by offering a means of owning and being able to tell one's story. Young people not only learn to process and integrate the traumatic experience into the larger context of their lives but are also empowered through the process of writing, narrating, illustrating, and ultimately assembling their own story. In doing so, they have the storytelling and technical tools to form adaptive responses regarding their family violence experiences, both in its immediate aftermath and over time, possibly preventing the trauma cycle from continuing.

\section{References}

Anderson, K. M. (2010). Enhancing resilience in survivors of family violence. NY: Springer.

Anderson, K. M., \& Hiersteiner, C. (2008). Recovering from childhood sexual abuse: Is a storybook ending possible? The American Journal of Family Therapy, 36(5), 413424.

Anderson, K. M., \& Wallace, B. (2015). Digital storytelling as a trauma narrative intervention for children exposed to domestic violence. In J. Cohen \& L. Johnson (Eds.), Film and video-based therapy (pp. 95-107). New York: Taylor \& Francis/Routledge.

Center for Digital Storytelling. (n.d.). Retrieved from http://storycenter.org 
Cohen, J. A., Mannarino, A. P., \& Deblinger, E. (2006). Treating trauma and traumatic grief in children and adolescents. NY: Guilford Press.

Cohen, J. A., Mannarino, A. P., \& Iyengar, S. (2011). Community treatment of posttraumatic stress disorder for children exposed to domestic violence: A randomized control trial. Archives of Pediatrics and Adolescent Medicine, 165(1), 1621.

Davis, A., \& Weinshenker, D. (2012). Digital storytelling and authoring identity. In C. C. Ching \& B. Foley (Eds.), Technology and identity: Research on the development and exploration of selves in a digital world (pp. 47-64). Cambridge, UK: Cambridge University Press.

Digital Storytelling. (2015). What is digital storytelling therapy? Retrieved from http://digitalstorytherapy.com/

Dube, S. R., Anda, R. F., Felitti, V. J., Edwards, V. J., \& Williamson, D. F. (2002). Exposure to abuse, neglect, and household dysfunction among adults who witnessed intimate partner violence as children: Implications for health and social services. Violence and Victims, 17(1), 3-17.

Foa, E. B., \& Rauch, S. A. (2004). Cognitive changes during prolonged exposure versus prolonged exposure plus cognitive restructuring in female assault survivors with posttraumatic stress disorder. Journal of Consulting Clinical Psychology, 72(5), 879884.

Foster, J. M. (2014). Supporting child victims of sexual abuse: Implementation of a trauma narrative family intervention. The Family Journal: Counseling and Therapy for Couples and Families, 22, 332-338.

Gubrium, A. (2009). Digital storytelling: An emergent method for health promotion research and practice. Health Promotion Practice, 10(2), 186-191.

Hanney, L., \& Kozlowska, K. (2002). Healing traumatized children: Creating illustrated storybooks in family therapy. Family Process, 41(1), 37-65.

Kress, G. (2003). Literacy in the new media age. London: Routledge.

Lambert, J. (2006). Digital storytelling: Capturing lives, creating community. Berkeley, CA: Digital Diner Press.

Niederhoffer, K. G., \& Pennebaker, J. W. (2009). Sharing one's story: On the benefits of writing or talking about emotional experience. In S. J. Lopez \& C. R. Snyder (Eds.), Oxford handbook of positive psychology (2nd ed., pp. 621-632). NY: Oxford University Press.

Polkinghorne, D. E. (1991). Narrative and self-concept. Journal of Narrative and Life History, 1(2 \& 3), 135-153.

Simon, V. A., Feiring, C., \& McElroy, S. K. (2010). Making meaning of traumatic events: Youths' strategies for processing childhood sexual abuse are associated with psychosocial adjustment. Child Maltreatment, 15(3), 229-241. 
van Minnen, A., Wessel, I., Dijkstra, T., \& Roelofs, K. (2002). Changes in PTSD patients' narratives during prolonged exposure therapy: A replication and extension. Journal of Traumatic Stress, 15(3), 255-258.

\section{Author note}

Address correspondence to: Kim M. Anderson, PhD, LCSW, School of Social Work, University of Missouri, 705 Clark Hall, Columbia, Missouri, 65211. Email: andersonki@missouri.edu. 